\title{
Detecting differences in volume pulse wave parameters among fingers and toes in four different postures
}

\author{
Tingting Yan, Lin Yang*, Song Zhang, Yimin Yang and Xuwen Li \\ College of Life Science and Bio-engineering, Beijing University of Technology, Beijing 100124, China
}

\begin{abstract}
The measurement of volume pulse waves may be easily affected based on the measured position (different fingers or toes), body posture, as well as other factors. Therefore, interferences during measurement need to be removed. For this study, relevant parameters of volume pulse wave were obtained by changing the affecting factors. The data were analyzed in order to indicate the differences in volume pulse wave parameters among different fingers and toes in four postures. Results showed that when subjects were lying, inclined or in a sitting posture, volume pulse wave parameters of the index finger, middle finger and ring finger on both hands displayed no apparent differences. When subjects were in a sitting posture, volume pulse wave parameters of the big, second and third toes on both feet showed no significant differences.
\end{abstract}

Keywords: Photoplethysmography, volume pulse wave parameters, different postures

\section{Introduction}

In recent years, photoplethysmography (PPG) has been utilized as a new method for noninvasive hemodynamic testing due to its simple operation, stable performance, and good reproducibility. Volume pulse wave is widely used to monitor oxygen saturation in clinical practices [1], which aids in the detection of peripheral blood circulation functions. Furthermore, it is used in noninvasive detection of blood flow parameters, as well as heart rate variability [2-4]. Professor Luo introduced a parameter of pulse wave named $K$ [5]. Through $K$, pulse waves can be used to calculate cardiovascular parameters noninvasively [6]. The characteristic $K^{\prime}$ of the volume pulse wave can be derived from the algorithm of the characteristic $K$ of the pressure of the pulse wave.

Characteristic $K^{\prime}$ represents a parameter of the volume pulse wave, defined as follows, where $Q_{\max }$ is the maximum value of the blood flow curve, $Q_{\min }$ is the minimum value of the blood flow curve, $Q_{\mathrm{m}}$ is the average value of the blood flow curve:

$$
K^{\prime}=\frac{\mathrm{Q}_{\mathrm{m}}-\mathrm{Q}_{\min }}{\mathrm{Q}_{\max }-\mathrm{Q}_{\min }}
$$

\footnotetext{
* Address for correspondence: Lin Yang, College of Life Science and Bio-engineering, Beijing University of Technology, No. 100 Ping le yuan, Chaoyang District, Beijing, China. Tel.: +8617090139526; Fax: +8667392010; E-mail: yanglin@bjut.edu.cn.
} 
The characteristic parameters $K_{1}^{\prime}$ and $K_{2}^{\prime}$ is based on separating the dicrotic wave trough, defined as follows [7], where $Q_{m l d}$ is the average value of the preceding blood flow curve of dicrotic wave trough, $Q_{m 2 d}$ is the average value of the latter part.

$$
\begin{aligned}
& K_{1}{ }^{\prime}=\frac{Q_{\operatorname{m1d}}-Q_{\min }}{Q_{\max }-Q_{\min }} \\
& K_{2}{ }^{\prime}=\frac{Q_{\operatorname{m2d}}-Q_{\min }}{Q_{\max }-Q_{\min }}
\end{aligned}
$$

$K^{\prime}, K_{1}^{\prime}$ and $K_{2}^{\prime}$ can represent the changes in some important physiological parameters such as peripheral vascular resistance and blood viscosity $[8,9]$. However, if these parameters of volume pulse wave are impacted by measured positions (different fingers and toes) or different measured postures is yet to be studied. Therefore, it is necessary to exclude the confounding factors from monitoring volume pulse wave.

\section{Methods and materials}

The experimental subjects and protocols were approved by the Institutional Research Review Board of Beijing University of Technology. For this study, 41 volunteers were observed, 25 male and 16 female between the ages of 20 to 25 and 32 cases of effective data were collected.

The experiment was separated into 2 parts; observing volume pulse wave parameters of different fingers (toes) in the same fixed posture and analyzing the same four parameters of the same fixed finger (toe) in different postures.

Subjects needed to remain stationary and keep calm with both hands kept relaxed on the sides of the body before the experiments. Approximately 5 minutes later, the volume pulse wave was monitored by a transmission photoelectric sensor. Since the little finger of most people are too thin to fit the transmission photoelectric sensor, only the thumb, index finger, middle finger and ring finger of both hands were chosen to be monitored while the test subject was in four different measurement postures; lying, inclined, sitting and standing. The methods were the same for the experiment on the different toes; however, because some toes are too short to wear transmission photoelectric sensor, only the big, second and third toes of both feet were chosen to be monitored.

\section{Results}

\subsection{Analyses of volume pulse wave parameters of different fingers in different postures}

Statistical analyses ANOVA tests were conducted. The results showing the differences among the

\begin{tabular}{|c|c|c|c|c|}
\hline \multicolumn{5}{|l|}{$\mathrm{P}_{\mathrm{K}}, \mathrm{P}_{\mathrm{K} 1}, \mathrm{P}$} \\
\hline & $\mathrm{P}_{\mathrm{K}}$, & $\mathrm{P}_{\mathrm{K} 1}$, & $\mathrm{P}_{\mathrm{K} 2}$, & $\mathrm{P}_{\text {amplitude }}$ \\
\hline Lying & 0.087 & 0.583 & 0.107 & 0.000 \\
\hline Inclined & 0.011 & 0.275 & 0.000 & 0.001 \\
\hline Sitting & 0.176 & 0.417 & 0.038 & 0.006 \\
\hline Standing & 0.000 & 0.000 & 0.000 & 0.000 \\
\hline
\end{tabular}

Table 1 
volume pulse wave parameters from the test subjects' fingers while in the same postures are shown in Table 1. It shows that fingers in fixed measured postures $\left(\mathrm{P}_{\mathrm{K}}, \mathrm{P}_{\mathrm{K} 1}, \mathrm{P}_{\mathrm{K} 2}\right.$, and $\left.\mathrm{P}_{\text {amplitude }}\right)$ did not exhibit measurements less than 0.05 .

By observing pulse waveforms of every proposed finger, results showed that the pattern of the pulse waveform of the thumbs was slightly different from the other fingers. Therefore, the experimental data of the thumbs were excluded from the final analyses. Mean values of $K^{\prime}, K_{1}{ }^{\prime}, K_{2}{ }^{\prime}$, amplitude of volume pulse wave at proposed fingers and different postures are shown in Table 2. This time, the test subjects were in fixed postures, excluding standing postures. Results showed that the $\mathrm{P}_{\mathrm{K}}, \mathrm{P}_{\mathrm{K} 1}, \mathrm{P}_{\mathrm{K} 2}$, and $\mathrm{P}_{\text {amplitude }}$ were less than 0.05 simultaneously, which can be found in Table 3. The results indicated that all volume pulse wave parameters were not statistically significant in different proposed fingers when the subjects remained in a lying, inclined or sitting posture.

Standing posture is rarely used for monitoring pulse waves. Furthermore, it was difficult to maintain a standing posture during the experiment. In order to exclude the error introduced during standing, only lying, inclined and sitting postures were analyzed. The results are shown in Table 4.

Table 2

Mean value of $K^{\prime}, K_{1}^{\prime}, K_{2}$ ' and amplitude of pulse wave in different fingers and postures

\begin{tabular}{|c|c|c|c|c|c|}
\hline Parameter & Finger & Lying & Inclined & Sitting & Standing \\
\hline \multirow[t]{6}{*}{$K^{\prime}$} & L2 & $0.47 \pm 0.05$ & $0.49 \pm 0.05$ & $0.51 \pm 0.06$ & $0.49 \pm 0.05$ \\
\hline & $\mathrm{R} 2$ & $0.47 \pm 0.06$ & $0.49 \pm 0.05$ & $0.52 \pm 0.04$ & $0.47 \pm 0.07$ \\
\hline & L3 & $0.47 \pm 0.04$ & $0.49 \pm 0.04$ & $0.50 \pm 0.05$ & $0.49 \pm 0.05$ \\
\hline & R3 & $0.47 \pm 0.05$ & $0.48 \pm 0.05$ & $0.50 \pm 0.05$ & $0.45 \pm 0.04$ \\
\hline & L4 & $0.47 \pm 0.05$ & $0.49 \pm 0.05$ & $0.50 \pm 0.04$ & $0.48 \pm 0.06$ \\
\hline & $\mathrm{R} 4$ & $0.47 \pm 0.05$ & $0.48 \pm 0.05$ & $0.50 \pm 0.03$ & $0.46 \pm 0.04$ \\
\hline \multirow[t]{6}{*}{$K 1^{\prime}$} & L2 & $0.67 \pm 0.06$ & $0.69 \pm 0.07$ & $0.71 \pm 0.05$ & $0.68 \pm 0.06$ \\
\hline & $\mathrm{R} 2$ & $0.68 \pm 0.07$ & $0.67 \pm 0.07$ & $0.69 \pm 0.05$ & $0.65 \pm 0.07$ \\
\hline & L3 & $0.68 \pm 0.05$ & $0.70 \pm 0.06$ & $0.70 \pm 0.06$ & $0.69 \pm 0.05$ \\
\hline & R3 & $0.68 \pm 0.05$ & $0.67 \pm 0.06$ & $0.69 \pm 0.04$ & $0.65 \pm 0.07$ \\
\hline & L4 & $0.68 \pm 0.06$ & $0.70 \pm 0.05$ & $0.69 \pm 0.06$ & $0.69 \pm 0.05$ \\
\hline & $\mathrm{R} 4$ & $0.68 \pm 0.07$ & $0.69 \pm 0.07$ & $0.70 \pm 0.05$ & $0.65 \pm 0.04$ \\
\hline \multirow[t]{6}{*}{$K 2^{\prime}$} & L2 & $0.32 \pm 0.07$ & $0.35 \pm 0.05$ & $0.38 \pm 0.07$ & $0.36 \pm 0.06$ \\
\hline & $\mathrm{R} 2$ & $0.32 \pm 0.07$ & $0.35 \pm 0.06$ & $0.39 \pm 0.06$ & $0.34 \pm 0.08$ \\
\hline & L3 & $0.33 \pm 0.06$ & $0.34 \pm 0.05$ & $0.37 \pm 0.06$ & $0.37 \pm 0.07$ \\
\hline & $\mathrm{R} 3$ & $0.32 \pm 0.05$ & $0.35 \pm 0.05$ & $0.37 \pm 0.07$ & $0.33 \pm 0.47$ \\
\hline & L4 & $0.33 \pm 0.08$ & $0.34 \pm 0.05$ & $0.37 \pm 0.06$ & $0.36 \pm 0.08$ \\
\hline & $\mathrm{R} 4$ & $0.32 \pm 0.05$ & $0.34 \pm 0.05$ & $0.38 \pm 0.06$ & $0.33 \pm 0.05$ \\
\hline \multirow{6}{*}{$\begin{array}{l}\text { amplitude } \\
\text { of volume } \\
\text { pulse wave }\end{array}$} & $\mathrm{L} 2$ & $109 \pm 39$ & $97 \pm 36$ & $89 \pm 46$ & $75 \pm 38$ \\
\hline & $\mathrm{R} 2$ & $103 \pm 46$ & $96 \pm 40$ & $78 \pm 37$ & $73 \pm 32$ \\
\hline & L3 & $105 \pm 46$ & $95 \pm 35$ & $99 \pm 43$ & $86 \pm 39$ \\
\hline & R3 & $116 \pm 53$ & $116 \pm 54$ & $86 \pm 42$ & $92 \pm 37$ \\
\hline & L4 & $111 \pm 53$ & $104 \pm 40$ & $92 \pm 42$ & $84 \pm 40$ \\
\hline & $\mathrm{R} 4$ & $114 \pm 55$ & $110 \pm 50$ & $92 \pm 53$ & $86 \pm 39$ \\
\hline
\end{tabular}

Table 3

$\mathrm{P}_{\mathrm{K}}, \mathrm{P}_{\mathrm{K} 1}, \mathrm{P}_{\mathrm{K} 2}, \mathrm{P}_{\mathrm{amplitude}}$ of index fingers, middle fingers and ring fingers of both hands in fixed measured postures

\begin{tabular}{lllll}
\hline & $\mathrm{P}_{\mathrm{K}}$, & $\mathrm{P}_{\mathrm{K} 1}$, & $\mathrm{P}_{\mathrm{K} 2}$, & $\mathrm{P}_{\text {amplitude }}$ \\
\hline Lying & 0.991 & 0.985 & 0.874 & 0.867 \\
Inclined & 0.901 & 0.341 & 0.899 & 0.300 \\
Sitting & 0.616 & 0.929 & 0.740 & 0.546 \\
Standing & 0.006 & 0.005 & 0.143 & 0.314 \\
\hline
\end{tabular}


Table 4

$\mathrm{P}_{\mathrm{K}}, \mathrm{P}_{\mathrm{K} 1}, \mathrm{P}_{\mathrm{K} 2}, \mathrm{P}_{\text {amplitude }}$ of different postures (except for standing position) in the same finger

\begin{tabular}{lllll}
\hline & $\mathrm{P}_{\mathrm{K}}$, & $\mathrm{P}_{\mathrm{K} 1}$, & $\mathrm{P}_{\mathrm{K} 2}$, & $\mathrm{P}_{\text {amplitude }}$ \\
\hline Right Index finger & 0.001 & 0.389 & 0.000 & 0.052 \\
Right middle finger & 0.079 & 0.259 & 0.000 & 0.026 \\
Right ring finger & 0.009 & 0.586 & 0.000 & 0.214 \\
Left Index finger & 0.016 & 0.078 & 0.004 & 0.136 \\
Left middle finger & 0.062 & 0.379 & 0.006 & 0.649 \\
Left ring finger & 0.036 & 0.305 & 0.065 & 0.217 \\
\hline
\end{tabular}

Different measurement postures had a greater impact on $K^{\prime}, K_{2}^{\prime}$, indicating that the postures had large influence on volume pulse waveforms. However, there was little influence by different postures on $K_{l}{ }^{\prime}$ and pulse wave amplitude. When only $K^{\prime}$ needs to be used in clinical application, middle fingers of both hands can be chosen to monitor in different postures. When $K_{1}{ }^{\prime}$ and pulse wave amplitude need to be analyzed, the right middle finger and ring fingers of both hands can be chosen.

\subsection{Analysis of volume pulse wave parameters of different toes in different postures}

According to single-factor analysis of variance, the differences of $K^{\prime}, K_{1}{ }^{\prime}, K_{2}{ }^{\prime}$ from proposed toes under the same postures are shown in Table 5. During a sitting posture, $\mathrm{P}$ values of $K^{\prime}, K_{1}{ }^{\prime}, K_{2}{ }^{\prime}$ for different toes were larger than 0.05 , which means there were no significant differences in parameters of proposed toes when subjects sit. However, results differed when subjects were in other postures.

In order to study the influence of different measurement postures on volume pulse wave parameters of different toes, the single-factor analysis of variance method was used to analyze the data, and results are shown in Table 6.

Different postures greatly impacted $K^{\prime}, K_{1}{ }^{\prime}, K_{2}{ }^{\prime}$ pulse wave amplitude. Results showed that the same toe in different measured postures had a $\mathrm{P}_{\text {amplitude }}=0.000$. There were some differences in measured postures between fingers and toes; in that toes couldn't be at the same horizontal position as the heart. Due to the influence of the potential energy, pulse wave amplitude from the same toe had significant differences in different postures. Therefore, this parameter was excluded from the four parameters of volume pulse wave analyses. In summary, the $\mathrm{P}$ value of $K^{\prime}, K_{1}{ }^{\prime}, K_{2}{ }^{\prime}$ for the right big toe were larger

Table 5

$\mathrm{P}_{\mathrm{K}}, \mathrm{P}_{\mathrm{K} 1}, \mathrm{P}_{\mathrm{K} 2}, \mathrm{P}_{\text {amplitude }}$ of the big, second, and third toes of both feet in fixed measured postures

\begin{tabular}{lllll}
\hline & $\mathrm{P}_{\mathrm{K}^{\prime}}$ & $\mathrm{P}_{\mathrm{K} 1^{\prime}}$ & $\mathrm{P}_{\mathrm{K}^{\prime}}$ & $\mathrm{P}_{\text {amplitude }}$ \\
\hline lying & 0.000 & 0.153 & 0.000 & 0.588 \\
Inclined & 0.026 & 0.305 & 0.027 & 0.702 \\
Sitting & 0.885 & 0.973 & 0.993 & 0.099 \\
\hline
\end{tabular}

Table 6

$\mathrm{P}_{\mathrm{K}}, \mathrm{P}_{\mathrm{K} 1}, \mathrm{P}_{\mathrm{K} 2}, \mathrm{P}_{\text {amplitude }}$ of the same toe in different measured postures

\begin{tabular}{lllll}
\hline & $\mathrm{P}_{\mathrm{K}}$, & $\mathrm{P}_{\mathrm{K} 1}$, & $\mathrm{P}_{\mathrm{K} 2}$, & $\mathrm{P}_{\text {amplitude }}$ \\
\hline Right big toe & 0.281 & 0.120 & 0.361 & 0.000 \\
Right second toe & 0.002 & 0.192 & 0.000 & 0.000 \\
Right third toe & 0.521 & 0.168 & 0.010 & 0.000 \\
left big toe & 0.002 & 0.001 & 0.269 & 0.000 \\
left second toe & 0.019 & 0.011 & 0.000 & 0.000 \\
left third toe & 0.574 & 0.085 & 0.009 & 0.000 \\
\hline
\end{tabular}


than 0.05 at the same time, if only $K^{\prime}, K_{1}{ }^{\prime}$ needed to be obtained, which demonstrated that the third toes of both feet also can be chosen.

\section{Discussion}

This study showed that when subjects were keeping the same posture, parameters of different fingers had significant differences. The differences were mainly caused by the mean value of $K^{\prime}, K_{1}{ }^{\prime}$, $K_{2}$ 'obtained from both thumbs which were larger than the other fingers due to the microcirculation differences between the thumb and the other fingers.

There were differences between monitoring fingers and toes, because toes couldn't be at the same horizontal level with the heart when subjects sitting or standing. The blood is impacted by gravity, producing hydrostatic pressure. When subjects were kept in a lying posture, the vascular system throughout the human body are basically at the same level, therefore, the hydrostatic pressure is approximately the same. However, when subjects were inclined, sitting or standing, the blood pressure in the foot was significant higher than subjects in a lying posture. This phenomena and its effect on the volume pulse wave in different measurement postures needs to be further studied.

\section{Conclusion}

In summary, There were significant differences in $K^{\prime}, K_{1}{ }^{\prime}, K_{2}{ }^{\prime}$ pulse wave amplitude when choosing different fingers (toes) and different measurement postures to detect volume pulse waves. When subjects were in a lying posture, inclined posture or sitting posture, index fingers, middle fingers and ring fingers of both hands could be chosen to monitor volume pulse waves. When subjects were in a sitting posture, the big, second and third toes of both feet could be chosen. This experimental study proposed to define a standard for detecting volume pulse waves in clinical applications.

\section{Acknowledgments}

This work is supported by the National Natural Science Foundation of China (No. 61374015).

\section{References}

[1] D.P. Bernstein, Pressure pulse contour-derived stroke volume and cardiac output in the morbidly obese patient, Obesity Surgery 18 (2008), 1015-1021.

[2] A. John, Photoplethysmography and its application in clinical physiological measurement, Physiological Measurement 28 (2007), 1-39.

[3] J.S. Yvonne, H. Inga and H. Imme, Arterial stiffness and pulse wave reflection are increased in patients suffering from severe periodontitis, Plos One 9 (2014), 1-8.

[4] Q.Y. Lee, S.J. Redmond and G.S.H. Chan, Estimation of cardiac output and systemic vascular resistance using a multivariate regression model with features selected from the finger photoplethysmogram and routine cardiovascular measurements, Biomedical Engineering Online 12 (2013), 1-16.

[5] W.W. Wang, L. Yang and S. Zhang, Clinical test and verify of hemodynamic parameters based on the volume pulse wave hemodynamic detecting, International Symposium on Information Technology in Medicine and Education, Changzhou, China, 2011, pp. 520-522.

[6] L. Yang, S. Zhang and Y. Yang, Study of pulse wave velocity noninvasive detecting instrument based on radial artery and 
finger photoplethysmography pulse wave, International Symposium on Intelligent Information Technology Application Workshops, Shanghai, China, 2008, pp. 705-708.

[7] L. Yang, S. Zhang and Y.M. Yang, Analysis of pulse contour characteristic values based on dicrotic wave trough, Beijing Biomedical Engineering 3 (2008), 229-233.

[8] J.L. Zhang, C.Y. Lin and L. Yang, Detection of waveform characteristic of pulse wave and analysis of its related partial hemodynamic changes, Biomedical Engineering and Clinical Medicine 2 (2008),104-107.

[9] L. Jin, L. Yang and S. Zhang, Computation of cardiac output by pulse wave contour, 1st International Conference on Bioinformatics and Biomedical Engineering, Wuhan, China, 2007, pp. 1088-1090. 